\title{
In vitro and in vivo growth inhibition of drug-resistant ovarian carcinoma cells using a combination of cisplatin and a TRAIL-encoding retrovirus
}

\author{
FANG LI $^{1,2 *}$, YI GUO ${ }^{1}$, LINGFEI HAN $^{1 *}$, YANKANG DUAN $^{1}$, FANG FANG $^{1}$, SUMEI NIU $^{1}$, \\ QIUJIE BA ${ }^{1}$, HUAISHI ZHU ${ }^{3}$, FANFEI KONG ${ }^{1}$, CHAO LIN ${ }^{2}$ and XUEJUN WEN ${ }^{2}$ \\ ${ }^{1}$ Cervical Disease Centre, Shanghai First Maternity and Infant Hospital, Tongji University School of Medicine, Shanghai \\ 200040; ${ }^{2}$ The Institute for Advanced Materials and Nano Biomedicine, Tongji University, Shanghai 200092; \\ ${ }^{3}$ Department of Radiology, Huangpu District Central Hospital, Shanghai 200002, P.R. China
}

Received June 22, 2012; Accepted August 31, 2012

DOI: $10.3892 / \mathrm{ol} .2012 .926$

\begin{abstract}
Retroviruses encoding the TNF-related apoptosisinducing ligand (TRAIL) gene were generated by transient transfection of the retrovirus packing cell line BOSC 23 using TRAIL-encoding plasmid. The retrovirus was able to transduce drug-resistant A2780/DDP ovarian carcinoma cells in vitro and induce TRAIL expression in the cells, as detected by western blot assay. Furthermore, the TRAIL protein led to the growth inhibition of the cells via a caspase-activated apoptotic mechanism. It was confirmed that exposure of such cells to cisplatin in combination with the TRAIL-encoding retrovirus resulted in higher anticancer activity in vitro and in the xenograft A2780/DDP tumor in a nude mouse model. This study suggests that chemotherapy in combination with TRAIL gene therapy may be an efficient approach to treat drug-resistant ovarian cancer.
\end{abstract}

\section{Introduction}

Ovarian carcinoma is the fifth most common gynecological cancer (1). Due to the lack of an efficient method for early diagnosis of ovarian cancer, the cancer is often detected in an advanced stage. In this situation, the patients usually have to undergo surgry to remove the tumor and receive chemotherapy using anticancer drugs, such as cisplatin and doxorubicin. In a number of cases, chemotherapy is, however, often discontinued due to intrinsic or developed drug resistance of ovarian

Correspondence to: Dr Fang Li, Cervical Disease Center, Shanghai First Maternity and Infant Hospital, Tongji University School of Medicine, 536 Changle Road, Jing'an, Shanghai 200040, P.R. China

E-mail: 09lifang@tongji.edu.cn

\section{${ }^{*}$ Contributed equally}

Key words: ovarian cancer, full length TRAIL, retrovirus, gene therapy, chemotherapy cancer cells (2-4). In order to obtain improved anticancer efficacy, chemotherapy in combination with a biological therapy has been studied in the past decade. This type of combined therapy was demonstrated to be more efficacious than either chemotherapy or biological therapy alone. For instance, a combination of an anticancer drug and trastuzumab, a monoclonal antibody capable of interfering with the HER2/neu receptor, led to enhanced growth suppression of breast cancer cells compared with anticancer drug alone (5). Besides, a previous study suggested that a combined use of anticancer drug with the TNF-related apoptosis-inducing ligand (TRAIL) protein had a synergistic effect on the apoptotic activity in ovarian cancer cells (6).

TRAIL is a promising anticancer protein which is capable of inducing specific apoptosis in a broad range of cancer cells, but not in normal cells (7-9). However, the clinical use of TRAIL protein is seriously impeded due to its instability in vivo and the resistance of cancer cells to the TRAIL $(10,11)$. To obtain favorable tumor growth inhibition, a high dose of the protein has to be administrated in vivo (10). As an alternative approach, virus-mediated TRAIL gene therapy has been investigated recently (12). This is attributed to the fact that viruses are able to transfer genes into somatic cells, thereby yielding highly efficient gene transfection. On the basis of this principle, Griffith et al constructed a TRAIL-encoded adenovirus and found that the virus may lead to TRAIL expression in cancer cells and to their death mediated by caspase-8-activated apoptosis (13). Furthermore, another study showed that an adenovirus-TRAIL may augment apoptosis in TRAIL-resistant cancer cells, including SKOV-3 ovarian cancer cells (14). These data indicate that virus-mediated TRAIL gene therapy may be promising for ovarian cancer therapy.

Recently, a few studies have been published on the chemotherapy drug in combination with TRAIL gene therapy for efficacious cancer therapy. For example, a previous study by Pan et al illustrated that a combined use of cisplatin with an oncolytic adenovirus-TRAIL resulted in synergistic growth inhibition of several types of cancer cell lines (15). Several other studies have proposed that chemotherapy drugs, such as 
cisplatin, may sensitize TRAIL-induced cytotoxicity in breast cancer cells or glioma cells, both in vitro and in vivo $(16,17)$. However, to the best of our knowledge, it is not well understood whether the combination of chemotherapy and TRAIL gene therapy can be applied to drug-resistant ovarian carcinoma cells.

In this study, we generated a recombinant retrovirusencoding TRAIL gene via TRAIL-plasmid transfection of the retrovirus packaging cell line BOSC 23. This virus was then used for the transduction of drug-resistant A2780/DDP ovarian carcinoma cells. The expression of the TRAIL gene in the cells was detected by western blot assay. The effect of the TRAIL on the in vitro growth of the retrovirus-transduced A2780/DDP cells was studied. Also, the growth-inhibitory effect of cisplatin in combination with the retrovirus-TRAIL was investigated in the xenograft A2780/DDP tumor in a nude mouse model.

\section{Materials and methods}

Cell culture. Retrovirus packaging BOSC 23 cells were cultured in the GPT-selective medium as previously described (18). Cisplatin-resistant A2780/DDP cells were maintained in DMEM (Gibco, Carlsbad, CA, USA) with 10\% fetal calf serum.

Recombinant retroviral vectors. Human TRAIL cDNA was kindly provided by Dr H. Yagita (Juntendo University School of Medicine, Japan). MSCV-IRES2-GFP plasmid was a gift from Dr R. Davis (University of Massachusetts, MA, USA). MSCV-TRAIL-IRES2-GFP plasmid was constructed by subcloning the TRAIL cDNA into the EcoRI site of the MSCV-IRES2-GFP plasmid as previously described (19).

Construction of retrovirus-TRAIL. Retrovirus-TRAIL was generated by calcium phosphate-mediated transient transfection of retrovirus packaging BOSC 23 cells using MSCV-TRAIL-IRES2-GFP plasmid (20). Specifically, BOSC 23 cells $\left(2.6 \times 10^{6}\right.$ cells $)$ were plated in a $60-\mathrm{mm}$ dish for at least $24 \mathrm{~h}$. Prior to cell transfection, cell culture medium was replaced with GPT-selective medium containing chloroquine $(50 \mu \mathrm{M})$. Subsequently, the cells were incubated with the coprecipitate of calcium phosphate and the plasmid $(8 \mu \mathrm{g})$ for $8 \mathrm{~h}$ at $37^{\circ} \mathrm{C}$ in a humidified $5 \% \mathrm{CO}_{2}$-containing atmosphere. Following this, the cell culture medium was replaced with fresh DMEM-complete medium and the cells were incubated for a further $16 \mathrm{~h}$. Expression of GFP was detected by Nikon fluorescence microscopy. Finally, viral supernatant was collected after centrifugation at $1,200 \mathrm{rpm}$ for $5 \mathrm{~min}$ at $4^{\circ} \mathrm{C}$ and stored at $-20^{\circ} \mathrm{C}$. Retrovirus-GFP without encoding TRAIL was also produced by the same procedure as mentioned above and used as a negative control. Viral titer was determined by flow cytometry (Beckton-Dickinson, San Jose, CA, USA) as previously described (19).

Construction of retrovirus-TRAIL-transduced A2780/DDP cells. A2780/DDP cells $\left(1 \times 10^{4}\right.$ cells per well) were plated in a 96-well plate with $200 \mu 1$ DMEM-complete medium containing $10 \%$ fetal calf serum for at least $24 \mathrm{~h}$. Then, the medium was replaced with viral supernatant $(125 \mu \mathrm{l})$, poly- brene $(4 \mu \mathrm{g} / \mathrm{ml})$ and fresh cytokines. After $24 \mathrm{~h}$ of infection, the cells were washed and resorted for GFP expression using FACScan. Individual clones showing a high level of GFP expression were selected by dilution cloning. Briefly, after flow cytometry analysis, pools of A2780/DDP cells were collected and plated in a 96-well plate with one to three cells per well. After incubation for several days, a single colony was identified under light microscopy and then further cultured in a $35-\mathrm{mm}$ dish. The clones that demonstrated a high level of GFP expression were used for further study. Retrovirus-GFPtransduced A2780/DDP cells without expressing TRAIL were also constructed by the same procedure as mentioned previously and used as a negative control.

MTT assay. Retrovirus-TRAIL-transduced, retrovirus-GFPtransduced A2780/DDP cells and untreated A2780/DDP cells as a control $\left(1.0 \times 10^{4}\right.$ cells per well) were seeded into a 96 -well plate. Cell survival, determined by MTT assay, was studied as a function of incubation time from 1 to 5 days. At different time intervals (days 1 to 5), MTT solution $(20 \mu 1,5 \mathrm{mg} / \mathrm{ml})$ was added to each well and incubated with the cells for $4 \mathrm{~h}$. Afterwards, the medium was removed and DMSO (150 $\mu \mathrm{l})$ was added to each well. The absorbance was measured at $570 \mathrm{~nm}$ using a plate reader (Thermo Scientific, Logan, UT, USA). The percentage of cell viability was determined relative to control cells (untreated A2780/DDP cells), and taken as $100 \%$ cell viability. All cytotoxicity experiments were performed eight times.

Western blot analysis. Retrovirus-TRAIL-transduced or retrovirus-GFP-transduced A2780/DDP cells were cultured in the absence or presence of caspase inhibitor zVAD-fmk for 3 days, then lysed and protein was extracted. Total protein was quantified by BCA protein assay kit (Promega Corporation, Madison, WI, USA). For western blot analysis, equal amounts of protein were separated by SDS-PAGE, transferred onto nitrocellulose membranes (Millipore, Bedford, MA, USA), blocked and incubated overnight with mouse monoclonal primary antibody. After washing, the membrane was incubated with horseradish peroxidaselabeled anti-mouse secondary antibody. Finally, the bands were visualized using the enhanced chemiluminescence kit (Amersham Biosciences) according to the manufacturer's instructions.

Apoptosis analysis. Sub-G1 proportion assays were performed to confirm apoptosis in A2780/DDP cells by retrovirusTRAIL. Briefly, retrovirus-TRAIL-transduced A2780/DDP cells, retrovirus-GFP-transduced A2780/DDP cells and untreated A2780/DDP cells (control) were cultured. After $48 \mathrm{~h}$, the cells were stained according to the manufacturer's instructions (CycleTest plus kit, Becton-Dickinson). The apoptotic cells were determined by counting the sub-G1 portion using a FACScan (Beckton-Dickinson) and the percentage of apoptotic cells was analyzed with CELL-Quest Version 3.3 software.

Animal study. Nude mice were housed in the animal facility at Tongji University. All research was approved by the Institutional Animal Care and Use Committee at the University. 
In order to generate tumors, $50 \mu \mathrm{l}$ of $2 \times 10^{6}$ single-cell suspension in 5\% PBS was injected subcutaneously into the backside of 4- to 6-week Balb/c nude mice of four groups ( $\mathrm{n}=6$ for each group). A2780/DDP cells were used in groups 1 (control) and 2 and retrovirus-TRAIL-transduced A2780/DDP cells were used in groups 3 and 4 . Treatment of tumors started at day 14 when the size of the tumors reached $800-1,000 \mathrm{~mm}^{3}$. At day 14, the chemotherapy drug cisplatin (CDDP) was administered to those mice of groups 2 and 4 . The tumor size was measured at day 40 , using a caliper across its longest (a) and shortest (b) diameters, and was calculated by the following equation: $\mathrm{V}=0.5 \mathrm{a} \mathrm{x}^{2}$.

Statistical analysis. Statistical differences between the experimental data of two groups were analyzed using a Student's t-test. Data were considered to be statistically significant at $\mathrm{P}<0.05$.

\section{Results}

Production of high-titer helper-free retrovirus expressing TRAIL. In this study, retroviruses that are encoded by TRAIL genes (retrovirus-TRAIL) were produced by calcium phosphate-mediated transfection of retrovirus packaging BOSC 23 cells using a TRAIL-GFP-encoded plasmid. This plasmid contains GFP gene reporter as an indicator of cell transfection. Prior to the transfection, coprecipitates of calcium phosphate/TRAIL-plasmid were formed in situ by drop-wise addition of the plasmid/ $\mathrm{CaCl}_{2}$ solution into the cell culture medium. After $16 \mathrm{~h}$ of transfection, hypertrophy was observed for the BOSC 23 cells (Fig. 1A), indicating the cellular internalization of the coprecipitates. After $48 \mathrm{~h}$ of transfection, 80-90\% BOSC 23 cells expressing GFP were observed under fluorescence microscopy (Fig. 1B). Eventually, the retroviruses were collected from the supernatant of the cell solution. Typical titer of the retrovirus was determined to be $1.96 \pm 0.37 \times 10^{6} \mathrm{TU} / \mathrm{ml}(19)$.

TRAIL led to the growth inhibition of drug-resistant A2780/ DDP cells via caspase-activated apoptosis. Drug-resistant A2780/DDP ovarian cancer cells were transduced by the retrovirus-TRAIL and an individual clone showing a high level of GFP expression was sorted with flow cytometry. The protein from the cell lysate was separated with SDS-PAGE and immunoblotted for TRAIL. A 32-kDa protein band was observed (Fig. 2). The size of the protein was consistent with that of TRAIL in a transmembrane form (13). Cell viability of retrovirus-TRAIL-transduced A2780/DDP cells as a function of incubation time was determined by MTT assay (Fig. 3). It was shown that cell viability significantly decreased from $83 \pm 7.2 \%$ to $36 \pm 6.7 \%$ when increasing the time from 1 to 5 days. By contrast, no cytotoxicity was observed for retrovirus-GFPtransduced or untreated A2780/DDP cells (Fig. 3).

TRAIL protein may induce apoptosis in cancer cells by a caspase-dependent mechanism (20). To ascertain whether TRAIL-induced apoptosis in A2780/DDP cells was due to triggering of the caspase cascade, caspase- 8 and PARP cleavage products in the cells were detected by western blot assay. As shown in Fig. 4, caspase- 8 was activated by the cleavage of a $55 / 53-\mathrm{kDa}$ precursor into $43 / 41-\mathrm{kDa}$ products. Activated
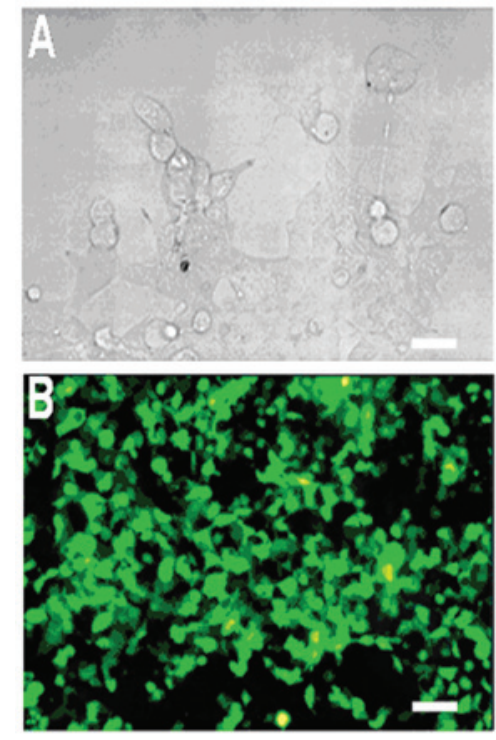

Figure 1. Calcium phosphate-mediated gene transfection of retrovirus packing cell line BOSC 23 using IRES-TRAIL-GFP plasmid. (A) Hypertrophy of BOSC 23 cells after $16 \mathrm{~h}$ of transfection. (B) GFP expression in BOSC 23 cells observed under fluorescence microscopy after $48 \mathrm{~h}$ of transfection. (scale bar, $20 \mu \mathrm{m}$ ). TRAIL, TNF-related apoptosis-inducing ligand.

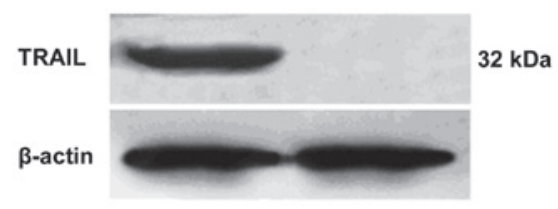

Figure 2. Western blot assay detected TRAIL protein in retrovirus-TRAILtransducted A2780/DDP cells. TRAIL, TNF-related apoptosis-inducing ligand.

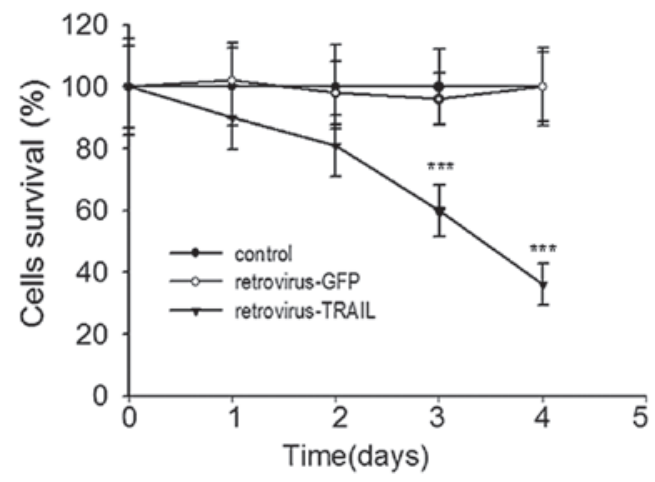

Figure 3. Cell viability of retrovirus-TRAIL-transduced A2780/DDP cells as a function of time. Retrovirus-TRAIL induced strong growth suppression compared with retrovirus-GFP or control cells. Data are the means of eight independent experiments. ${ }^{* * *} \mathrm{P}<0.001$, retrovirus-TRAIL vs. retrovirus-GFP. TRAIL, TNF-related apoptosis-inducing ligand.

caspase- 8 then cleaved PARP from a $116-\mathrm{kDa}$ protein into an $85-\mathrm{kDa}$ cleavage product. Besides, the presence of caspase inhibitor zVAD-fmk resulted in the loss of these cleavage products in the cells. As a negative control, these cleavage products were absent from the retrovirus-GFP-transduced A2780/DDP cells. 


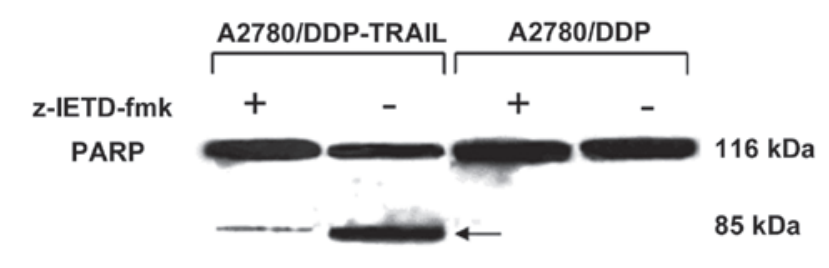

procaspase-8

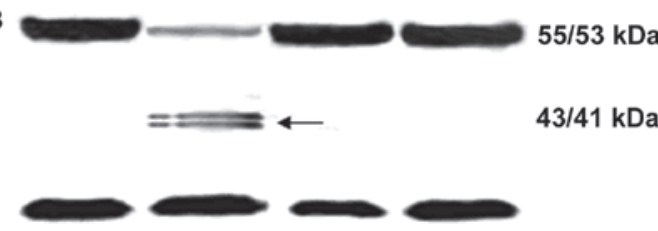

Figure 4. Western blot assay detected TRAIL protein in retrovirus-TRAILtransduced A2780/DDP cells in the absence and presence of caspase-8 inhibitor z-IETD-fmk $(50 \mu \mathrm{M})$. Caspase-8 activation was detected due to intermediate cleavage products of 43/4-kDa from 55/53-kDa native forms. The 116-kDa PARP and its $85-\mathrm{kDa}$ cleavage product are indicated by arrows. TRAIL, TNF-related apoptosis-inducing ligand.

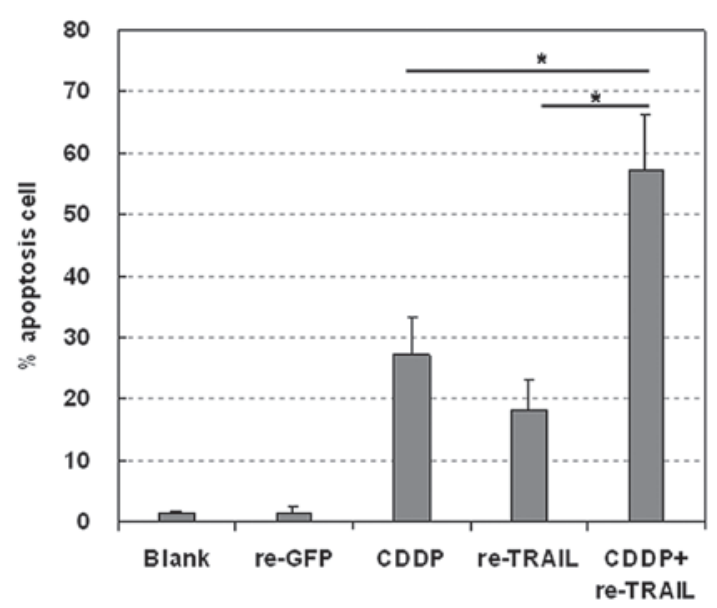

Figure 5. Cisplatin (CDDP) in combination with retrovirus-TRAIL led to enhanced apoptosis in A2780/DDP cells as compared with CDDP or retrovirus-TRAIL (re-TRAIL) alone. Retrovirus-GFP (re-GFP) was used as a negative control. The percentage of apoptotic cells was determined using flow cytometry. "P<0.05 (data are the means of three independent experiments). TRAIL, TNF-related apoptosis-inducing ligand.

Cisplatin in combination with retrovirus-TRAIL may augment apoptosis in A2780/DDP cells. Effects of the retrovirusTRAIL, alone or in combination with cisplatin, on the growth of the A2780/DDP cells were studied in vitro. The percentage of apoptotic cells was detected with sub-G1 proportion assay and determined by flow cytometry (Fig. 5). Retrovirus-TRAIL and cisplatin resulted in $18.3 \pm 5.0 \%$ and $27.3 \pm 5.9 \%$ apoptotic cells, respectively. By contrast, exposure of such cells to the retrovirus-TRAIL and cisplatin induced a higher percentage of apoptotic cells $(57.2 \pm 9.2 \%)$.

Retrovirus-TRAIL inhibits A2780/DDP tumor growth in a nude mouse model. A preliminary in vivo study was performed to evaluate the antitumor activity of retrovirusTRAIL. Tumors were established by subcutaneous injection of either A2780/DDP cells or retrovirus-TRAIL-transduced
Table I. Growth inhibition of drug-resistant A2780/DDP cells inoculated in nude mice.

\begin{tabular}{|c|c|c|}
\hline Group no. & Treatment & Tumor volume $\left(\mathrm{mm}^{3}\right)$ \\
\hline 1 & Control & $3375 \pm 23.3$ \\
\hline 2 & CDDP & $1301 \pm 21.0$ \\
\hline 3 & Retrovirus-TRAIL & $729 \pm 10.1$ \\
\hline 4 & Retrovirus-TRAIL + CDDP & $27 \pm 1.7^{\mathrm{a}}$ \\
\hline
\end{tabular}

The mean sizes of tumors are presented as mean \pm SD $(n=6)$. ${ }^{\mathrm{a}} \mathrm{P}<0.001$, group no. 4 vs. no. 2 (or 3 ). CDDP, cisplatin; TRAIL, TNF-related apoptosis-inducing ligand.

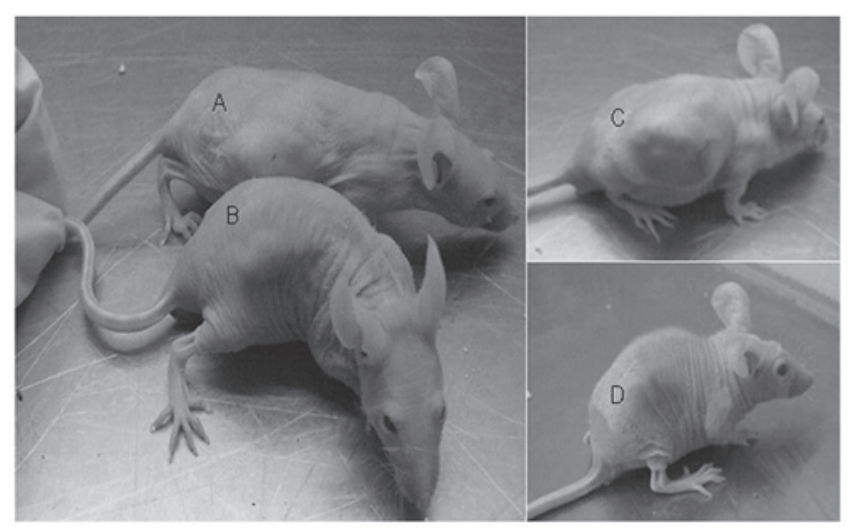

Figure 6. The mean size of tumor from retrovirus-transduced A2780/ DDP cells (A) was smaller than that of tumor from A2780/DDP cells (D), after CDDP administration at day 40. (A) Tumor from A2780/DDP cells treated with CDDP, (B) tumor from retrovirus-TRAIL-transduced A2780/ DDP, (C) tumor from A2780/DDP as a control, (D) tumor from retrovirusTRAIL--transduced A2780/DDP cells treated with CDDP. CDDP, cisplatin; TRAIL, TNF-related apoptosis-inducing ligand.

A2780/DDP cells into the backside of nude mice. A2780/DDP cells formed appreciable tumors at day 10 in all mice. By contrast, retrovirus-TRAIL-transduced A2780/DDP cells formed visible tumors at a later time (day 12) in 4 out of 6 nude mice. At day 14, anticancer drug cisplatin was administrated to these mice via i.v. injection. Tumor size was measured at day 40 (Fig. 6; Table I). The tumor size of retrovirus-TRAIL-treated A2780/DDP cells was smaller than that of untreated A2780/DDP cells alone $\left(729 \pm 10.1\right.$ vs. $\left.3375 \pm 23.3 \mathrm{~mm}^{3}\right)$. It was also found that the combination treatment of the A2780/DDP tumor with cisplatin and retrovirus-TRAIL resulted in the highest antitumor activity $\left(27 \pm 1.7 \mathrm{~mm}^{3}\right)$.

\section{Discussion}

TNF-related apoptosis-inducing ligand (TRAIL), also known as Apo2L, is a type II integral membrane protein belonging to the TNF family. It is a promising agent for cancer therapy owing to its capability of inducing apoptosis in numerous cancer cell lines, but little adverse effect on most normal cells. However, clinical use of TRAIL protein is seriously limited due to its instability in vivo and the resistance of cancer cells. TRAIL gene therapy provides an alternative approach. Viral vectors 
are potent to transfer therapeutic genes into the cancer cells, thus resulting in a high level of gene expression. Adenoviral vectors carrying the TRAIL gene were reported that may lead to apoptosis in cancer cells and a bystander effect (14).

In this study, we sought to investigate the possibility of chemotherapy in combination with TRAIL gene therapy for drug-resistant ovarian cancer. Firstly, TRAIL-encoded retrovirus was generated by calcium phosphate-mediated TRAIL-plasmid transient transfection of the retrovirus packing cell line BOSC 23 (Fig. 1). In a previous study, hightiter retrovirus was produced from the retrovirus packing cell line by the transient gene transfection (20). As a result, retroviruses carrying TRAIL gene were generated with a high titer $\left(1.96 \times 10^{6} \mathrm{TU} / \mathrm{ml}\right)$. To the best of our knowledge, this is the first report on the production of TRAIL-encoded retrovirus.

Western blot analysis showed that TRAIL protein $(32-\mathrm{kDa})$ was produced in the drug-resistant A2780/DDP cells transduced by the retrovirus-TRAIL (Fig. 2), suggesting the expression of TRAIL gene in the cells. Moreover, the TRAIL protein resulted in significant growth inhibition of the A2780/DDP cells, when the transduced cells were cultured from 1 to 5 days (Fig. 3). It was expected that the appreciable growth suppression may be due to TRAIL-mediated cell apoptosis via a caspase-activated mechanism. As evidence of this, caspase- 8 and PARP cleavage products in retrovirus-TRAIL-transduced A2780/DDP cells were detected by western blot assay. The results in Fig. 4 indicate that A2780/DDP growth inhibition by retrovirus-TRAIL may be due to the induction of caspase- 8 activated apoptosis. Support for this hypothesis was also found in the western blot obtained in the presence of caspase inhibitor zVAD-fmk. As expected, the inhibitor efficiently led to the loss of these cleavage products. These data are in line with the results from Seol et al who revealed that TRAIL protein is capable of inducing caspase-8-dependent apoptosis in Jurkat cells (22).

The combinatory effect of cisplatin with retrovirus-TRAIL was studied in A2780/DDP cells in vitro and in vivo. The in vitro study showed that cisplatin in combination with the retrovirus-TRAIL led to a higher percentage of apoptotic cells compared with retrovirus-TRAIL or cisplatin alone (Fig. 5). Furthermore, a preliminary in vivo study revealed that the combination treatment of A2780/DDP tumor with cisplatin and the retrovirus-TRAIL induced the highest antitumor activity (Fig. 6; Table I). These data suggest that a combination of chemotherapy and TRAIL-gene therapy may be an efficient approach to killing drug-resistant ovarian cancer cells.

We have demonstrated that high-titer, TRAIL-encoded retrovirus may be generated by TRAIL-plasmid transfection of retrovirus packing cells BOSC 23. The retroviruses were able to transduce drug-resistant A2780/DDP ovarian carcinoma cells and to induce the TRAIL-gene expression and their growth inhibition in vitro via caspase-8-activated apoptosis. Moreover, the chemotherapy drug cisplatin, in combination with retrovirus-TRAIL, may lead to augmented growth inhibition of A2780/DDP cells in vitro and of the xenograft A2780/DDP tumor in a nude mouse model.

\section{Acknowledgements}

This study was supported by grants from the Health Bureau of Shanghai (No.20114150), Innovation Program (No.
11QA1405200) and National Natural Science Foundation of China (No. 81001153).

\section{References}

1. Landis SH, Murray T, Bolden S and Wingo PA: Cancer statistics, 1999. CA Cancer J Clin 49: 8-31, 1999.

2. Kaufmann SH and Earnshaw WC: Induction of apoptosis by cancer chemotherapy. Exp Cell Res 256: 42-49, 2001.

3. Fisher DE: Apoptosis in cancer therapy: crossing the threshold. Cell 78: 539-542, 1994.

4. Schmitt CA and Lowe SW: Apoptosis is critical for drug response in vivo. Drug Resist Updat 4: 132-134, 2001.

5. Baselga J, Norton L, Albanell J, Kim YM and Mendelsohn J: Recombinant humanized anti-HER 2 antibody (Herceptin) enhances the antitumor activity of paclitaxel and doxorubicin against HER2/neu overexpressing human breast cancer xenografts. Cancer Res 58: 2825-2831, 1998.

6. Cuello M, Ettenberg SA, Nau MM and Lipkowitz S: Synergistic induction of apoptosis by the combination of TRAIL and chemotherapy in chemoresistant ovarian cancer cells. Gynecol Oncol 81: 380-390, 2001.

7. Ferreira CG, Epping M, Kruyt FA and Giaccone G: Apoptosis: target of cancer therapy. Clin Cancer Res 8: 2024-2034, 2002.

8. Sheridan JP, Marsters SA, Pitti RM, et al: Control of TRAIL-induced apoptosis by a family of signaling and decoy receptors. Science 277: 818-821, 1997.

9. Ashkenazi A: Targeting death and decoy receptors of the tumournecrosis factor superfamily. Nat Rev Cancer 2: 420-430, 2002.

10. Walczak H, Miller RE, Ariail K, et al: Tumoricidal activity of tumor necrosis factor related apoptosis-inducing ligand in vivo. Nat Med 5: 157-163, 1999.

11. Grotzer MA, Eggert A, Zuzak TJ, et al: Resistance to TRAIL-induced apoptosis in primitive neuroectodermal brain tumor cells correlates with a loss of caspase- 8 expression. Oncogene 19: 4604-4610, 2000.

12. Ma H, Liu Y, Liu S, Kung HF, Sun X, Zheng D and Xu R: Recombinant adeno-associated virus-mediated TRAIL gene therapy suppresses liver metastatic tumor. Int J Cancer 2: 314-321, 2005.

13. Griffith TS, Anderson RD, Davidson BL, Williams RD and Ratliff TL: Adenoviral-mediated transfer of the TNF-related apoptosis-inducing ligand/apo-2 ligand gene induces tumor cell apoptosis. J Immunol 165: 2886-2894, 2000.

14. Seol JY, Park KH, Hwang CI, et al: Adenovirus-TRAIL can overcome TRAIL resistance and induce a bystander effect. Cancer Gene Ther 10: 540-548, 2003.

15. Pan Q, Liu B, Liu J, Cai R, Wang Y and Qian C: Synergistic induction of tumor cell death by combining cisplatin with an oncolytic adenovirus carrying TRAIL. Mol Cell Biochem 304: 315-323, 2007.

16. Keane MM, Ettenberg SA, Nau MM, Russell EK and Lipkowitz S: Chemotherapy augments TRAIL-induced apoptosis in breast cell lines. Cancer Res 59: 734-741, 1999.

17. Nagane M, Pan G, Weddle JJ, Dixit VM, Cavenee WK and Huang HJ: Increased death receptor 5 expression by chemotherapeutic agents in human gliomas causes synergistic cytotoxicity with tumor necrosis factor-related apoptosis-inducing ligand in vitro and in vivo. Cancer Res 60: 847-853, 2000.

18. Krosl J, Baban S, Krosl G, Rozenfeld S, Largman C and Sauvageau G: Cellular proliferation and transformation induced by HOXB4 and HOXB3 proteins involves cooperation with PBX1. Oncogene 16: 3403-3412, 1998.

19. Jiang QL, Wang JM, Jiang S, Wen LM and Zhou H: Large-scale real-time titration of green-fluorescence-protein-marked recombinant retrovirus: comparison with standard titration method. Di Yi Jun Yi Da Xue Xue Bao 23: 1101-1103, 2003 (In Chinese).

20. Pear WS, Nolan GP, Scott ML and Baltimore D: Production of high-titer helper-free retroviruses by transient transfection. Proc Natl Acad Sci USA 90: 8392-8396, 1993.

21. Kassis R, Larrous F, Estaquier J and Bourhy H: Lyssavirus matrix protein induces apoptosis by a TRAIL-dependent mechanism involving caspase-8 activation. J Virol 78: 6543-6555, 2004.

22. Seol DW, Li J, Seol MH, Park SY, Talanian RV and Billiar TR: Signaling events triggered by tumor necrosis factor-related apoptosis-inducing ligand (TRAIL): caspase- 8 is required for TRAIL-induced apoptosis. Cancer Res 61: 1138-1143, 2001. 